\title{
Experiments with heavy, highly charged ions - Status of the HITRAP project
}

\author{
F. Herfurth ${ }^{* * 1}$, W. Barth ${ }^{1}$, G. Clemente ${ }^{1}$, L. A. Dahl ${ }^{1}$, P. Gerhard ${ }^{1}$, M. Kaiser ${ }^{1}$, O. K. \\ Kester $^{1,2}$, H.-J. Kluge ${ }^{1}$, C. Krantz ${ }^{3}$, N. Kotovskiy ${ }^{1}$, C. Kozhuharov ${ }^{1}$, M. Maier ${ }^{1}$, D. \\ Neidherr $^{1}$, W. Quint ${ }^{1}$, U. Ratzinger ${ }^{2}$, A. Reiter ${ }^{1}$, A. Schempp ${ }^{2}$, A. Sokolov ${ }^{1}$, Th. \\ Stöhlker ${ }^{1}$, H. Vormann ${ }^{1}$, G. Vorobjev ${ }^{1}$, A. Wolf ${ }^{3}$ and S. Yaramishev ${ }^{1}$ for the HITRAP \\ collaboration \\ ${ }^{1}$ GSI Helmholtz Centre for Heavy Ion Research, Planckstraße 1, 64291 Darmstadt, Germany \\ ${ }^{2}$ Institute for Applied Physics, Goethe-Universität Frankfurt, Max-von-Laue-Str. 1, 60438 \\ Frankfurt am Main, Germany \\ ${ }^{3}$ Max-Planck-Institute for Nuclear Physics, Saupfercheckweg 1, 69117 Heidelberg Germany
}

\begin{abstract}
At the GSI accelerator complex, using the universal linear accelerator UNILAC and the synchrotron SIS, highly-charged ions up to $\mathrm{U}^{92+}$ are produced by passing a $400 \mathrm{MeV} / \mathrm{u}$ beam through a gold foil stripping off all or nearly all electrons. The HITRAP facility is built to decelerate those ions to almost rest and to provide them to experiments. In a number of commissioning beam times, the deceleration in the ESR, the extraction, bunching and deceleration to $0.5 \mathrm{MeV} / \mathrm{u}$ has been shown. The remaining steps, deceleration to $6 \mathrm{keV} / \mathrm{u}$ and cooling in a cryogenic Penning trap are ongoing. Precision experiments for atomic and nuclear physics purpose are being prepared and range from laser spectroscopy on stored ions, collision experiments with complete kinematic analysis to high precision mass measurements on single highly charged ions.
\end{abstract}

8th International Conference on Nuclear Physics at Storage Rings-Stori11,

October 9-14, 2011

Laboratori Nazionali di Frascati dell'INFN, Italy

\footnotetext{
* Speaker.

†F.Herfurth@gsi.de
} 


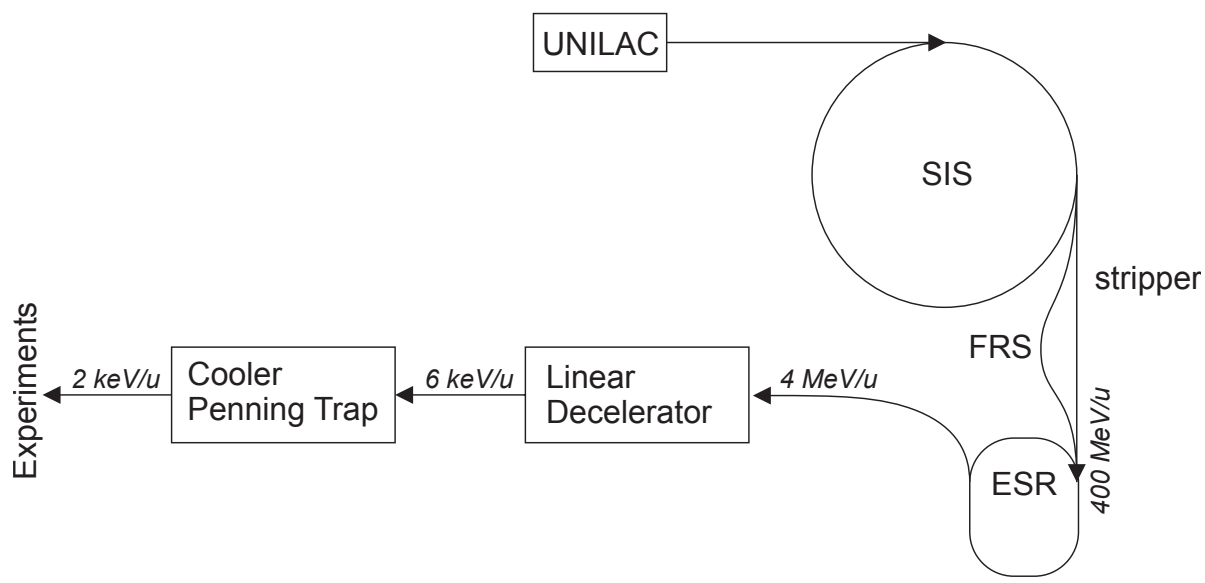

Figure 1: Overview of the HITRAP facility.

\section{Introduction}

Heavy, highly-charged ions, in the extreme case up to $\mathrm{U}^{91+}$ or even the bare $\mathrm{U}^{92+}$, are well suited for cutting edge experiments in atomic, nuclear and solid state physics [1]. They are simple but come along with a very strong electric field due to the heavy nucleus. This supplies the testing ground for the appropriate field theory, quantum electrodynamics (QED), at the strong-field limit. Measurable quantities for those tests are the g-factor of the bound electron, the electron binding energies or the hyperfine splitting. For those measurements, which require high precision to be decisive, the ions have to be stored, as single ion or in an ion cloud, in a well defined environment at very low energy as offered by a Penning trap. The observation of the stored particles will then allow for mass measurements at the ppt level and hence the binding energies of the components with $\mathrm{eV}$ precision, the determination of the bound state g-factor with a precision that even tests our knowledge of fundamental constants like the mass of the electron or, combined with laser excitation several hundred times more precise investigation of the transition energies between hyperfine levels than feasible before [2].

Also, yet unseen reaction phenomena can be explored: Heavy, highly-charged ions are very instable systems when in close contact with electrons since a huge potential energy is concentrated in a very small volume. When those HCI at very low energy come close to neutral matter relaxation processes happen very fast and give snapshot-like insight into the dynamics and correlation of the electrons in the neutral collision partner. If energy and position are well defined the exchange of multiple charges can be studied by a complete analysis of the kinematics of all involved particles. For that, highly-charged ions are accumulated in a Penning trap and cooled by electron and resistive cooling to about $4 \mathrm{~K}$ [3]. After ejection a well defined ion beam will be targeted to a cold sample of neutral atoms and the products will be investigated by a reaction microscope [4]. Two different target types will be applied for HITRAP experiments, a pulsed gas target and a magneto optical trap.

When the large potential energy is concentrated and released on a small spot on the surface of a solid, self-ordering phenomena have been observed and need further investigation to clarify the 


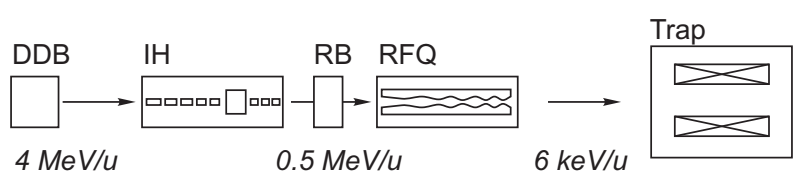

Figure 2: The functional units of the HITRAP linear decelerator: the double drift buncher (DDB), the interdigital H-type structure (IH), a spiral re-buncher (RB), the four-rod radio frequency quadrupole structure (RFQ) and the cooler Penning trap (Trap).

role of impact energy and potential energy. This can be accomplished only if the impact energy, i.e. the kinetic energy of the particle, is well below the potential energy of a few $100 \mathrm{keV}$ [5].

All those experiments require that kinetic energy and spatial position of the highly-charged ions can be well controlled. This is in contradiction to the most efficient production process that employs stripping of electrons at high energies by sending relativistic highly-charged ions with still many electrons through matter. The solution is a decelerator and storage facility for highly-charged ions produced by stripping all electrons of the ions from a $400 \mathrm{MeV} / \mathrm{u}$ beam - the HITRAP facility.

HITRAP is setup at the accelerator facility of the GSI Helmholtzcenter for Heavy Ion Research in Darmstadt/Germany (fig. 1). The universal linear accelerator UNILAC is used to deliver intermediate charged ions with $11 \mathrm{MeV} / \mathrm{u}$ to the heavy ion synchrotron SIS. After acceleration to $400 \mathrm{MeV} / \mathrm{u}$ the ions are send through a thin foil such that all electrons are stripped of the nucleus. The experimental storage ring ESR is then used to cool the such produced ion beam and decelerate it to $4 \mathrm{MeV} / \mathrm{u}$ in two steps. After initial stochastic cooling the beam is decelerated to $30 \mathrm{MeV} / \mathrm{u}$ and then cooled with electrons. The next deceleration step from $30 \mathrm{MeV} / \mathrm{u}$ to $4 \mathrm{MeV} / \mathrm{u}$ is followed by another electron cooling period before the coasting beam is bunched in $1 \mu \mathrm{s}$ bunches and ejected towards the HITRAP linear decelerator and facility.

The linear decelerator (fig. 2) consist of a double drift buncher at 108 and $216 \mathrm{MHz}$ for preparation of the beam to the longitudinal acceptance of the first decelerating structure, the interdigital $\mathrm{H}$-type structure (IH). There, the ions are decelerated from $4 \mathrm{MeV} / \mathrm{u}$ down to $500 \mathrm{keV} / \mathrm{u}$. An intermediate rebuncher shall ensure maximum efficiency when the beam is injected into the second decelerating structure, a four-rod radio frequency quadrupole decelerator (RFQ). Both decelerating structures run at $108 \mathrm{MHz}$ and require a power of 200 and $80 \mathrm{~kW}$, respectively. In the RFQ the ion beam is decelerated from $500 \mathrm{keV} / \mathrm{u}$ to $6 \mathrm{keV} / \mathrm{u}$ and then dynamically captured in a Penning trap for final cooling.

\section{Commissioning of the linear decelerator}

Funding for the construction of HITRAP has started in 2005 and commissioning of first components started 2007. About two commissioning beam times have been granted per year with an average duration of 6 days. An overview of those beam times and the achieved or planned steps is displayed in table 1.

Presently, the deceleration to $500 \mathrm{keV} / \mathrm{u}$ has been shown and is working efficiently [11]. The major breakthrough was the successful installation of an energy sensitive detector, which gives shot by shot the complete energy spectrum of the ions after the IH structure [10]. However, the 
Table 1: Overview of conducted commissioning beam times.

\begin{tabular}{rcl}
\hline Date & Ion & Comment \\
\hline $5-2007$ & ${ }^{64} \mathrm{Ni}^{28+}$ & Vacuum separation ESR/HITRAP installed - acceptance test \\
$8-2007$ & ${ }^{20} \mathrm{Ne}^{10+}$ & Double Drift Buncher test using diamond detector in front of IH \\
$8-2008$ & ${ }^{197} \mathrm{Au}^{79+}$ & Bunches transported through IH, analyzed on diamond det. [6] \\
$10-2008$ & ${ }^{64} \mathrm{Ni}^{28+}$ & First decelerated particles at 500 keV/u with energy spectrometer \\
$2-2009$ & ${ }^{58} \mathrm{Ni}^{28+}$ & First run after IH retuning. 4 MeV/u transport-mode test \\
$6-2009$ & ${ }^{132} \mathrm{Xe}^{54+}$ & Beam measured with pepper-pot emittance meter [7, 8, 9] \\
$3-2010$ & ${ }^{86} \mathrm{Kr}^{33+}$ & Improved IH energy analyzer [10] \\
$4-2010$ & ${ }^{86} \mathrm{Kr}^{35+}$ & Beam to RFQ, energy analysis sensitive only to 6 keV/u [11] \\
$11-2010$ & ${ }^{86} \mathrm{Kr}^{35+}$ & RFQ Energy analyzer also to 500 keV/u sensitive \\
$5-2011$ & ${ }^{54} \mathrm{Cr}^{24+}$ & IH Energy measurements \\
$6-2011$ & ${ }^{14} \mathrm{~N}^{7+}$ & IH Energy measurements, RFQ parameters scanned \\
$9-2011$ & ${ }^{136} \mathrm{Xe}^{50+}$ & IH and DDB combined optimisation, ESR energy scan \\
\hline
\end{tabular}

optimization of the combination of two bunchers (combined in the double drift buncher) and the IH is still difficult and not completely understood. The large parameter space given by three RF amplitudes and two phases requires the investigation of many different settings. This takes a lot of time and is virtually impossible due to the low repetition rate of at most one shot every 30 seconds. Two improvements have been implemented: the energy of the particles entering the ESR was limited to $30 \mathrm{MeV} / \mathrm{u}$ for commissioning of the linear decelerator which saves one deceleration step and hence time; the longitudinal analysis of the bunches created by the double drift buncher has been enhanced by a dedicated analysis procedure [12].

The focus of recent beam times was on the detailed investigation of the IH structure output. Supported by detailed simulations [13] the deceleration efficiency was increased and reached above $50 \%$, very close to the theoretical limit.

Unfortunately, all efforts to decelerate the beam further with the RFQ structure failed so far. The most probable reason is a slight mismatch of the IH output energy distribution and the energy range accepted by the RFQ. This was found in recently updated simulations of the RFQ structure made possible by a detailed 3D measurements of the electrodes. To verify those findings and work towards a solution the RFQ is being installed at a pelletron accelerator at the Max-Planck Institute for Nuclear Physics in Heidelberg/Germany. There, the energy can be easily varied in the desired range around $500 \mathrm{keV} / \mathrm{u}$ and there will be ample time available to measure the RFQ performance systematically.

The cooler Penning trap is tested offline with deuterium ions from a cross beam ion source. An electron source has been installed already to provide the electrons for the electron cooling scheme [14]. It is based of UV light released electrons from a GaAs surface and is fully compatible with the required ultra-high vacuum. Electrons have been captured already in the cooler Penning trap and the ion capture process is being tested in the moment. 


\section{Experimental installations}

Already in the original proposal several experimental stations where foreseen. Those are the g-factor setup to measure the g-factor of the bound electron in heavy, highly-charged ions; SPECTRAP for laser spectroscopy on cooled and trapped ion ensembles to measure the hyperfine structure [15]; a gas target to enable collision experiments analyzed by a reaction microscope; a Penning trap for precise mass measurements; a surface reaction chamber to investigate the dynamics during approach and the modifications induced when HCI approach surfaces [16]. Later, a magneto optical trap has been designed in Heidelberg (MOTRIMS) and constructed to serve as a target of very high density and hence increase the collision cross sections as well as the variety of available elements since gaseous targets are typically limited to rare gases.

The superconducting magnet for the g-factor trap has been installed and the trap design is finished. A number of electronic components like the detection circuit have been tested offline [17]. The spectroscopy trap will contain a small electron source to produce in trap test ions as for instance argon that is fed by a purpose designed cryogenic gas valve [18].

The SPECTRAP setup, the overhauled RETRAP [19], formerly installed at Livermore, is a Penning trap setup dedicated to laser cooling and spectroscopy. The trap has been reconstructed with special focus on laser and light detection access. In $2011, \mathrm{Mg}^{+}$ions could be stored, laser cooled and detected via both, their fluorescence, and their image charge signal [20].

Recently a small, room temperature electron beam ion trap (EBIT) has been installed to provide light and medium heavy, highly charged ions. It could be shown for the first time that charge breeding in such a small "Dresden" type source is possible and yields notable efficiencies [21]. This way, a multitude of elements can be delivered as test beam to the the experimental stations, including non gaseous ones, as for instance potassium or magnesium.

The beam line that connects the HITRAP cooler Penning trap to the experiments [22] and the EBIT has been completed and is now being commissioned offline using the EBIT ions send backwards to the cooler Penning trap. The further line towards SPECTRAP is being setup and will be finished by Spring 2012 .

\section{Summary and Outlook}

The HITRAP decelerator facility is well on its way for first experiments in 2012/13. The deceleration from $400 \mathrm{MeV} / \mathrm{u}$ down to $500 \mathrm{keV} / \mathrm{u}$ has been shown with sufficient efficiency. However, there is still room for improvement when it comes to the repetition rate as well as the optimal settings of the involved components, like the double drift buncher and the IH structure. The final deceleration step is being investigated since first tries did not yield positive results. For that a test bench has been setup at a dedicated $500 \mathrm{keV} / \mathrm{u}$ beam in Heidelberg and the characterization of the RFQ structure is ongoing there.

Most of the experiments are well on track too, they are being installed at the HITRAP facility or have proven to work elsewhere and wait for their transfer to the HITRAP facility. A test ion source for light and medium heavy highly charged ions of a multitude of elements has been installed at the HITRAP facility meanwhile. 
We would like to acknowledge the help of the accelerator, beam diagnostics and RF departments at GSI was well as at the MPI-K in Heidelberg. Special thanks goes to the mechanical workshop and the technology lab at GSI. Funding was provided in parts by the BMBF, the german ministry for education and research, as well as the European Commission.

\section{References}

[1] H.-J. Kluge et al., HITRAP: A Facility at GSI for Highly Charged Ions, Adv. Quant. Chem. 53 (2008), 83-98.

[2] M. Vogel, W. Quint, and W. Nörtershäuser, Trapped Ion Oscillation Frequencies as Sensors for Spectroscopy, Sensors 10 (2010), no. 3, 2169-2187.

[3] G. Maero et al., Numerical investigations on resistive cooling of trapped highly charged ions, Applied Physics B: Lasers and Optics (2011), 1-10.

[4] J. Ullrich et al., Recoil-ion momentum spectroscopy, Journal of Physics B: Atomic, Molecular and Optical Physics 30 (1997), no. 13, 2917.

[5] A. S. El-Said et al., Creation of Nanohillocks on $\mathrm{CaF}_{2}$ Surfaces by Single Slow Highly Charged Ions, Phys. Rev. Lett. 100 (2008), 237601.

[6] L. Dahl et al., The HITRAP Decelerator Project at GSI - Status and Commissioning Report, Proceedings of the XIV Linear Accelerator Conference - LINAC08, 2008, p. 102.

[7] J. Pfister et al., Commissioning of the HITRAP decelerator using a single-shot pepper-pot emittance meter, Proceedings of the XIV Linear Accelerator Conference, 2008, p. 564.

[8] F.Herfurth et al., The HITRAP Decelerator Linac at GSI, Proceedings of PAC09, Vancouver, BC, Canada, 2009, p. 1961.

[9] F. Herfurth et al., HITRAP - Heavy, Highly-Charged Ions and Antiprotons at Rest, Act. Phys. Pol. B 41 (2010), no. 2, 457.

[10] G. Vorobjev et al., Diagnostic Scheme for the HITRAP Decelerator, 10th European Workshop on Beam Diagnostics and Instrumentation for Particle Accelerators, 2011, p. 311.

[11] F. Herfurth et al., HITRAP - A Decelerator for Heavy, Highly-charged Ions, Proceedings of Linear Accelerator Conference LINAC2010, Tsukuba, Japan, 2010, p. 151.

[12] A. Reiter et al., in preparation.

[13] G. Clemente, private comm., 2011.

[14] Claude Krantz, Intense Electron Beams from GaAs Photocathodes as a Tool for Molecular and Atomic Physics, Ph.D. thesis, Ruprecht Karls Universität, Heidelberg, Germany, 2009.

[15] Z. Andjelkovic et al., Towards high precision in-trap laser spectroscopy of highly charged ions, Hyperfine Interactions 196 (2010), 81-91.

[16] E. Bodewits et al., Electron emission yields from boron-like Ar ions impinging on Au(100), Nucl. Instr. and Meth. B 269 (2011), no. 11, 1203 - 1207, Proceedings of the 18th International Workshop on Inelastic Ion-Surface Collisions (IISC-18).

[17] N. Brantjes et al., A Penning trap for g-factor measurements in highly charged ions by laser-microwave double-resonance spectroscopy, Hyperfine Interactions 199 (2011), 161-166. 
[18] D. von Lindenfels et al., Bound electron g-factor measurement by double-resonance spectroscopy on a fine-structure transition, Canadian Journal of Physics 89 (2011), no. 1, 79-84.

[19] D.A. Church et al., Collisions and spectroscopy of cold, highly charged high-Z ions in RETRAP, Nucl. Instr. and Meth. B 132 (1997), no. 2, 335 - 343.

[20] Z. Andjelkovic, Ph.D. thesis, Johannes Gutenberg Universität, Mainz, Germany, 2012, in preparation.

[21] A Sokolov et al., SPARC EBIT - a charge breeder for the HITRAP project, Journal of Instrumentation 5 (2010), no. 11, C11001.

[22] F. Herfurth et al., Precision measurements with highly charged ions at rest: The HITRAP project at GSI, International Journal of Mass Spectrometry 251 (2006), no. 2-3, 266 - 272. 